\title{
Finite Gravity: From the Big Bang to Dark Matter
}

\author{
Allen D. Allen \\ Physics Division, New Terra Enterprises, Glorieta, USA \\ Email: allend.allen@yahoo.com
}

Received February 27, 2013; revised March 30, 2013; accepted April 6, 2013

Copyright (C) 2013 Allen D. Allen. This is an open access article distributed under the Creative Commons Attribution License, which permits unrestricted use, distribution, and reproduction in any medium, provided the original work is properly cited.

\begin{abstract}
The purpose of the present paper is to assume that the expanding spacetime of our cosmos was created by the big bang. It then follows that there exists a finite instantaneous radial extent $d R_{U}$ to spacetime as observed from anywhere in spacetime by comoving observers. The consequences for gravity are explored by first considering the scalar field of a central mass that defines the dynamic properties of a circular orbit for each radius $R \leq d R_{U}$ under the postulate of weak equivalence. These properties include an orbital velocity and an escape velocity. For a central mass of galactic proportion, the escape velocity becomes large even at cosmological distances. By considering the dynamics of a smaller mass occupying the last orbit, we find that the established laws of physics lead to different rotation curves than they do when applied to the solar system. Since galactic rotation curves reveal the existence of dark matter, this is anticipated to have some consequences for our understanding of dark matter.
\end{abstract}

Keywords: Gravity; Weak Equivalence; Rotation Curves; Big Bang; Finite Gravity; Dark Matter

\section{Introduction}

Much has been learned since Kepler used the observations of Tyco Brahe to lay a foundation for Newtonian gravity, as subsequently embodied in general relativity through the correspondence principle. Yet the ancient concept of fixed, passive and infinite space still colors the rotation curves that play an important role in modern astronomy and astrophysics. This includes the rotation curves of spiral galaxies that led to the widespread acceptance of a poorly understood dark matter [1-3]. It also includes the rotation curves of stars orbiting the centers of galaxies suggesting that supermassive black holes reside in galactic nuclei $[4,5]$.

This short paper will admit to the dynamic spacetime of the big bang with an emphasis on the implications for rotation curves under the standard laws of physics.

\section{Formalities}

\subsection{The Central Mass}

Throughout this paper, $M$ will denote a large, isolated rest mass. By beginning with this mass and the two postulates below, we can explore the effect on gravity of having a gravitational field that has a finite instantaneous extent as measured from anywhere in spacetime by co- moing observers rather than extending to the traditional infinite limit.

\subsection{Postulates}

Weak Equivalence Postulate: Gravitational mass and inertial mass are equivalent.

Big Bang Postulate: Spacetime was created by the big bang and is expanding through finite radial extents $d R_{U}$.

Note that without the Big Bang Postulate, we could hardly state an estimated age for the cosmos as a single-valued function, as is a common practice.

\section{The Scalar Field}

It follows from the definition of the central mass $M$ and the Weak Equivalence Postulate that for each radial distance $R$ from the center of $M$, there exists uniquely a scalar field $\delta_{M R}$ that describes precisely the inherent, dynamic properties of the unoccupied circular orbit of radius $R$ :

$$
\widetilde{S}_{M R}=\left|\begin{array}{c}
I \\
v \\
\xi
\end{array}\right|=\left|\begin{array}{c}
-G M R^{-2} \\
\left(G M R^{-1}\right)^{1 / 2} \\
\left(2 G M R^{-1}\right)^{1 / 2}
\end{array}\right|
$$

where $I$ is the field intensity, $v$ is the orbital speed, and $\xi$ 
is the escape speed.

Recall that traditionally the field is deemed to end and free space to begin at the limit

$$
\begin{aligned}
& I \rightarrow 0 . \\
& R \rightarrow \infty
\end{aligned}
$$

But (2) is replaced here by the Big Bang Postulate, above. It follows from the Big Bang Postulate that there is a minimum escape speed.

$$
\varepsilon=\left(2 G M R_{U}^{-1}\right)^{1 / 2}
$$

Hence, there is a finite range $\boldsymbol{q}_{M}$ for $\varsigma_{M R}$,

$$
Я_{M}=2 G M \varepsilon^{-2} .
$$

It follows from (3) and (4) that

$$
Я_{M}=R_{U},
$$

which is virtually a tautology. The relativist should recognize that when a satellite orbits the central mass $M$, the satellite is travelling along the geodesic due to the curving of spacetime by the stress-energy tensor of $M$. Obviously, this is impossible beyond the range $R_{U}$ of the spacetime. Thus, substituting from (3) into (1), the smallest field intensity is finite,

$$
I(\varepsilon)=\varepsilon^{4}(4 G M)^{-1}
$$

On inspection of (1) it is obvious that escape speed $\xi$ is monotone increasing as mass,

$$
\xi \propto M^{1 / 2} .
$$

Otherwise, we would not have black holes. Table 1 shows some values for the minimum escape speed $\varepsilon$ as a function of mass, with $d R_{U}=14 \times 10^{9}$ light-years. Although the current value is uncertain [6], this is on the general order of a cosmological extent.

\section{The Gravitating System}

Let a satellite of mass $m \ll M$ occupy a circular orbit of $M$ with a radius of $R$. (By $m \ll M$ is meant that $m$ is

Table 1. Minimum escape speed $\varepsilon$ for various masses with a field range of $R_{U}=14 \times 10^{9}$ light-years.

\begin{tabular}{ccc}
\hline Mass in $\odot$ & $\varepsilon$ in $\mathbf{~} / \mathbf{s}$ & $\varepsilon$ in $\mathbf{k p h}$ \\
\hline 0.001 (Jovian) & $4.48 \times 10^{-5}$ & $1.61 \times 10^{-4}$ \\
1 & $1.41 \times 10^{-3}$ & $5.10 \times 10^{-3}$ \\
$1,000,000$ & 1.42 & 5.10 \\
$100,000,000$ & 14.16 & 50.10 \\
$1,000,000,000$ & 44.79 & 161.23 \\
$10,000,000,000$ & 141.63 & 509.86 \\
$2 \times 10^{11}$ & 633.38 & 2280 \\
\hline
\end{tabular}

sufficiently point-like compared to $M$ to orbit $M$ rather than their mutual center of mass.) Then there exists uniquely a force operator

$$
\mathscr{F}=[m \boldsymbol{i}, \boldsymbol{p}, \boldsymbol{j}],
$$

where $\boldsymbol{i}$ is the unit vector for the gravitational force, $\boldsymbol{p}$ is the unit vector for angular velocity, and $\boldsymbol{j}$ is the unit vector for the tangent to the orbit pointing away from $M$. The non-Albian product

$$
\mathscr{f} \Phi_{M R}=\left[-G M m R^{-2} \boldsymbol{i},\left(G M R^{-1}\right)^{1 / 2} \boldsymbol{p},\left(2 G M R^{-1}\right)^{1 / 2} \boldsymbol{j}\right] \text {. }
$$

is a gravitating system. From (1) and (9) the orbital velocity of the satellite of mass $m$ is

$$
v=\left(G M R^{-1}\right)^{1 / 2} .
$$

For $v \ll c$ we can write the kinetic energy of the satellite as

$$
T=\frac{1}{2} m v^{2} .
$$

Substituting from (10) into (11) we obtain

$$
T=\frac{1}{2} G M m R^{-1},
$$

such that

$$
\mathrm{d} T / \mathrm{d} R=-\frac{1}{2} G M m R^{-2}=\frac{1}{2} F,
$$

where we see from (9) that $F$ is the force of gravity. According to (12), the radius $R$ of the satellite's orbit expands and contracts in inverse proportion to the kinetic energy $T$ of the satellite,

$$
T \propto R^{-1}
$$

Thus, (14) conserves angular momentum.

It may be seen from Table 1 that when $M$ approaches galactic magnitude, its satellite becomes tightly bound. At $2 \times 10^{11}$ solar masses (on the order of the mass of the Milky Way) the satellite is so tightly bound to $M$ that the escape velocity is $2280 \mathrm{kph}$ even at the distance $d R_{U}$, equivalent to the full extent of spacetime. It is as if the central mass and its satellite were the endpoints of a rigid rod. Consequently, at this galactic scale, it is not (14) that conserves angular momentum but rather an invariant period of rotation, $\Delta t=2 \pi \operatorname{rad} / \theta=\mathrm{a}$ constant. Since $\varepsilon \propto\left(M d R_{U}^{-1}\right)^{1 / 2}$, this "dark matter" will evaporate as $d R_{U}$ continues to expand unless $M$ radiates away its rest mass.

\section{Conclusion}

We have considered a large, isolated, central rest mass $M$. If we accept weak equivalence, then for each radial dis- 
tance $R$ from $M$ there exists uniquely a scalar field $\varsigma_{M R}$. It describes the inherent, dynamic properties of an unoccupied circular orbit of $M$ with radius $R$. If we accept the big bang theory, then spacetime has a finite instantaneous radius $d R_{U}$, albeit monotone increasing as time, as measured from any point in spacetime by comoving observers. Hence, the escape speed in $\varsigma_{M R}$ has a minimum value of $\varepsilon=\left(2 G M d R_{U}{ }^{1}\right)^{1 / 2}$ and the range of $\varsigma_{M R}$ is the finite $d R_{U}$. Next we consider a satellite of mass $m \ll M$ that occupies a circular orbit of $\varsigma_{M R}$ with radius $R$. Then there exists uniquely a force operator $\mathrm{F}$, such that the non-Albian product $\mathcal{F}_{M R}$ is a gravitating system. The radius $R$ of the orbit in $\mathscr{F}_{M R}$ expands and contracts in inverse proportion to the kinetic energy $T$ of the satellite, $T \propto R^{-1}$. This conserves angular momentum. But as the central mass $M$ becomes galactic in magnitude, $M$ and the satellite become tightly bound. For $M=2 \times 10^{11}$ solar masses, the escape velocity is $2280 \mathrm{kph}$, even at the distance $d R_{U}$, equivalent to the full extent of spacetime. It is as if $M$ and $m$ were the endpoints of a rigid rod. Hence, at this galactic scale, angular momentum is conserved by a constant period of rotation $\Delta t=2 \pi \mathrm{rad} / \theta=$ a constant. Since $\varepsilon \propto\left(M d R_{U}^{-1}\right)^{1 / 2}$, this "dark matter" will evaporate as $d R_{U}$ continues to expand unless $M$ radiates away its rest mass.

\section{REFERENCES}

[1] K. G. Begeman, " $\mathrm{H}_{\mathrm{I}}$ Rotation Curves of Spiral Galaxies," Astronomy and Astrophysics, Vol. 223, No. 1-2, 1989, pp. 47-60.

[2] S. M. Kent, "Dark Matter in Spiral Galaxies. I. Galaxies with Optical Rotation Curves," Astrophysical Journal, Vol. 91, No. 6, 1986, pp. 1301-1327.

[3] T. S. van Albada, J. N. Bahcall, K. Begeman, and R. Sanscisi, "Distribution of Dark Matter in The Spiral Galaxy NGC 3198," Astrophysical Journal, Vol. 295, 1985, pp. 305-313.

[4] J. Kormendy and D. Richstone, "Inward Bound-The Search for Supermassive Black Holes in Galactic Nuclei," Annual Review of Astronomy and Astrophysics, Vol. 33, 1995, pp. 581-624.

[5] J. Kormendy, "Evidence for a Supermassive Black Hole in the Nucleus of M31," Astrophysical Journal, Vol. 325, 1988, pp. 128-141.

[6] B. M. S. Giambastiani, "Evoluzione Idrologica ed Idrogeologica Della Pineta di San Vitale (Ravenna)," Ph.D. Thesis, Bologna University, Bologna, 2007. 